International Journal of Modern Physics A

(C) World Scientific Publishing Company

\title{
Probing the QGP Phase Boundary with Thermal Properties of $\phi$ Mesons
}

\author{
Raghunath Sahoo ${ }^{1,2 *}$, Tapan K. Nayak ${ }^{3}$, Jan-e Alam ${ }^{3}$, Basanta K. Nandi ${ }^{4}$ and Sonja Kabana ${ }^{5}$ \\ ${ }^{1}$ Indian Institute of Technology Indore, Indore-452017, India \\ ${ }^{2}$ Dipartimento di Fisica dell'Università and Sezione INFN di Padova, Italy \\ ${ }^{3}$ Variable Energy Cyclotron Centre, Kolkata-700064, India \\ ${ }^{4}$ Indian Institute of Technology, Powai, Mumbai-400076, India \\ ${ }^{5}$ SUBATECH, 4, Rue Alfred Kastler, BP 20722 - 44307 Nantes, France
}

\begin{abstract}
A novel attempt has been made to probe the QCD phase boundary by using the experimental data for transverse momenta of $\phi$ mesons produced in nuclear collisions at AGS, SPS and RHIC energies. The data are confronted with simple thermodynamic expectations and lattice QCD results. The experimental data indicate a first order phase transition, with a mixed phase stretching the energy density between $\sim 1$ and $3.2 \mathrm{GeV} / \mathrm{fm}^{3}$ corresponding to SPS energies.
\end{abstract}

Keywords: Heavy-ion collisions, quark-gluon plasma, deconfinement transition

PACS numbers: 25.75.Nq, 12.38.Mh

\section{Introduction}

Numerical simulations of Quantum Chromodynamics (QCD) on lattice at finite temperature and density predict that at high temperature and/or energy density hadrons melt down to a new state of matter, called Quark Gluon Plasma (QGP) which might have existed after a few microseconds of the Big Bang. Lattice QCD based calculations $1|2| 3$ indicate that the phase transition from a hadronic state to a state of deconfined quarks and gluons occurs beyond a critical temperature $\left(T_{c}\right)$ and a critical baryonic chemical potential $\left(\mu_{c}^{B}\right)$. The order of the QCD phase transition and the properties of the QGP state have been a matter of intense debate, lately. According to the lattice QCD (1QCD) calculations, the order of the phase transition depends on the quark masses and more importantly, on the baryochemical potential $\left(\mu_{B}\right)$. These calculations suggest that the QCD phase diagram has a point in temperature-baryonic chemical potential $\left(T-\mu_{B}\right)$ plane where the first order phase transition ends. The exact location of this point in terms of $T$ and $\mu_{B}$ is not known yet, but the lQCD calculations suggest that it might be within the reach of heavy-ion experiments. Since the magnitude of the baryochemical potential at the central rapidity region depends on the collision energy (it reduces with the increase

*Corresponding author, e-mail: raghu@mail.cern.ch 
in beam energy), by scanning over a broad energy range, it would be possible to study the change in the nature of QCD phase transition along the phase boundary and probe the critical point.

A combination of several observables is required in order to study the quarkhadron phase boundary and to locate the QCD critical point. In this article, we point out that $\phi$ meson may be used as a sensitive probe for studying the phase boundary. The $\phi$ meson is the lightest bound state of hidden strangeness $(s \bar{s})$, and is produced early in the reaction. The $\phi$ meson is least suffered by hadronic rescattering. Relatively long lifetime $(\sim 45 \mathrm{fm} / \mathrm{c})$ of $\phi$ mesons may ensure that the decay products are least affected by re-scattering with other hadrons as the decay occurs outside the fireball 45 . On one hand, the theoretical calculations $\frac{617}{6}$ show different amount of modification of the $\phi$ meson properties in the medium due to its interaction with other mesons in the thermal bath. On the other hand, the results extracted from the analysis of the experimental data on the ratio of various hadronic

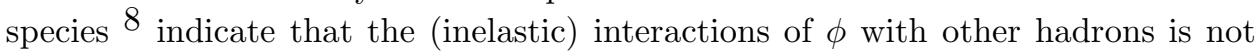
significant for temperatures below $T_{c}$. In view of the other systematic effects entering to the measurements of the transverse momentum distribution of $\phi$, the change in

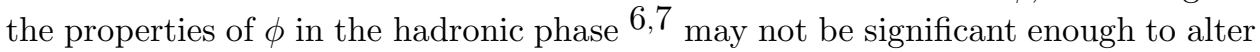
the results of the present analysis. The kinematic observables and flow phenomena of the $\phi$ mesons are particularly interesting for asserting the equation of state and the nature of the phase transition.

\section{Thermal Properties, Bjorken Energy density and Deconfinement Transition}

The initial energy density plays a very crucial role in the evolution of the fireball which is estimated as follows. The volume of a cylinder of radius $R$ and length $d z$ is given by $d V=\pi R^{2} d z=\pi R^{2} \tau \cosh y d \eta$ where $\tau=\sqrt{t^{2}-z^{2}}$, is the proper time and $\eta=\frac{1}{2} \ln \frac{t+z}{t-z}$, is the space-time rapidity. Identifying $\eta$ with the kinematic rapidity, $y$ and considering the volume element of width $d y$ in rapidity space around midrapidity $(y=0)$ at the initial time, $\tau_{0}$ one gets the value of the initial volume as $d V=\pi R^{2} \tau_{0}$. If the total initial energy within the rapidity window $d y$ is $d E_{T}$ then the initial energy density $\epsilon_{\mathrm{Bj}}$, as was first discussed in a seminal paper by Bjorken 9 is given by,

$$
\epsilon_{\mathrm{Bj}}=\left\langle\frac{d E_{\mathrm{T}}}{d y}\right\rangle \frac{1}{\pi R^{2} \tau_{0}}=\left\langle\frac{d N}{d y}\right\rangle\left\langle m_{\mathrm{T}}\right\rangle \frac{1}{\pi R^{2} \tau_{0}},
$$

where $d N / d y$ is the number of hadrons per unit rapidity and $\left\langle m_{\mathrm{T}}\right\rangle$ is the mean transverse mass. In the absence of detailed hydrodynamic calculations, this expression has been widely used by various experiments $10|11| 12 \mid 13$ to estimate the initial energy density. However, the Bjorken energy density given by Eqn. 1 could be much smaller than the actual initial energy density produced in nuclear collisions. The estimated energy density in the framework of Bjorken model may be taken as a lower 
bound $14[15$ due to strong reduction, when compared with the initially produced energy density from longitudinal hydrodynamic work during the expansion.

In a first order phase transition scenario, the pressure increases with temperature until the transition temperature $T_{c}$ is reached, then it remains constant during the mixed phase, and continues to increase after the end of the mixed phase. In a similar fashion, the deconfinement transition can be studied by observing the variation of average transverse momentum $\left(\left\langle p_{\mathrm{T}}\right\rangle\right)$ of hadrons as a function of the hadron multiplicity at mid-rapidity $(d N / d y) 16$. The $\left\langle p_{\mathrm{T}}\right\rangle$ is expected to reflect the average temperature $\left(T_{\mathrm{th}}\right)$ and a flow component which can be related to the initial pressure. Similarly the hadronic multiplicity reflects the entropy density of the system. While the purely thermal component of $\left\langle p_{\mathrm{T}}\right\rangle$ cannot be related to the initial temperature which remains immeasurable directly above $T_{c}$, however the flow component in the inverse slope can reflect the plateau of the pressure during mixed phase. It has been observed that in central heavy ion collisions, the $\left\langle m_{\mathrm{T}}\right\rangle\left(=\sqrt{p_{\mathrm{T}}^{2}+m^{2}}\right)$ of pions, kaons and protons as a function of $d N_{\mathrm{ch}} / d y$ shows a step-like behavior as predicted in 16 for a wide range of collision energies 17/18. Hydrodynamic calculations assuming a first order transition could reproduce these data 17/19. Here, we have made an attempt to make the study for $\phi$-mesons which could provide a better understanding of the QCD phase boundary, because of the nature of the interaction of $\phi$ with hadronic matter.

The transverse momentum $\left(p_{\mathrm{T}}\right)$ spectra of $\phi$-mesons measured for AGS 20121, SPS $22 \mid 23$ and RHIC 24/25 energies have been analyzed in order to obtain the inverse slope parameter, $T_{\text {eff }}$. This effective temperature has contributions from both the (random) thermal and the collective motions in the transverse direction. It is well known that the inverse slope $\left(T_{\text {eff }}\right)$ of the transverse momentum spectra of a hadron of mass $m$ can be related to the 'true' freeze-out temperature $\left(T_{\mathrm{th}}\right)$ and average radial flow velocity $\left.\left(<v_{\mathrm{r}}\right\rangle\right)$ at the decoupling surface as:

$$
T_{\text {eff }}=T_{\mathrm{th}}+\frac{1}{2} m\left\langle v_{\mathrm{r}}\right\rangle^{2},
$$

The transverse flow extracted from the $p_{\mathrm{T}}$ spectra of $\phi$ meson is particularly useful because of its small re-scattering cross-section with hadrons, the $\phi$ meson is expected to obtain most of its collective flow from the partonic phase which is produced in the early stages of heavy-ion collisions.

Because of long lifetime of $\phi$-meson it can probe the reaction time scales in a definite fashion. The $p_{\mathrm{T}}$ spectra of $\phi$-mesons may reveal quite a few information about the evolution of the system. These can be obtained by making a blast wave (BW) model description 26 of the experimental spectra. For this purpose, we have chosen data corresponding to only central collisions of $\mathrm{Au}-\mathrm{Au}$ or $\mathrm{Pb}-\mathrm{Pb}$ at different energies. Although the BW parametrization of the $m_{\mathrm{T}}$ spectra could give a set of values for $T_{\mathrm{e} f f}$ and $\left\langle v_{\mathrm{r}}\right\rangle$, we have taken the best possible values (with reduced $\left.\chi^{2} \leq 1\right)$ for our present studies. The results of the BW fit have been shown in Fig. 1 and the extracted values of $\left\langle v_{\mathrm{r}}\right\rangle$ and $T_{\mathrm{t} h}$ are listed in Table I. The table also 
enlists $T_{\text {eff }}$ and other basic parameters of different colliding systems at different collision energies. The similarity of the extracted $T_{\text {th }}$ to the critical temperature $T_{\text {c }}$ predicted by lattice calculations 1 imply that the $\phi$ meson freezes out near the phase boundary and could be used to extract the properties of QCD matter near the transition point. Moreover the large values of $v_{\mathrm{r}}$ at RHIC energies indicate that QGP has undergone substantial radial flow.

A compilation of measured data for central heavy ion collisions for $T_{\text {eff }}$, for low $p_{\mathrm{T}}$ range $\left(0<p_{\mathrm{T}} \leq 3.0 \mathrm{GeV} / \mathrm{c}\right)$ and at mid-rapidity has been made $20|21| 22|23| 24|25| 27|28| 29$ as shown in Table I. The results are depicted in Fig. 2 as a function of collision energy $\sqrt{s_{\mathrm{NN}}}$. Both the hadronic and leptonic decay channels of $\phi$-mesons are included in this figure. The $T_{\mathrm{th}}$ and $v_{r}$ in Eq. (2) have been
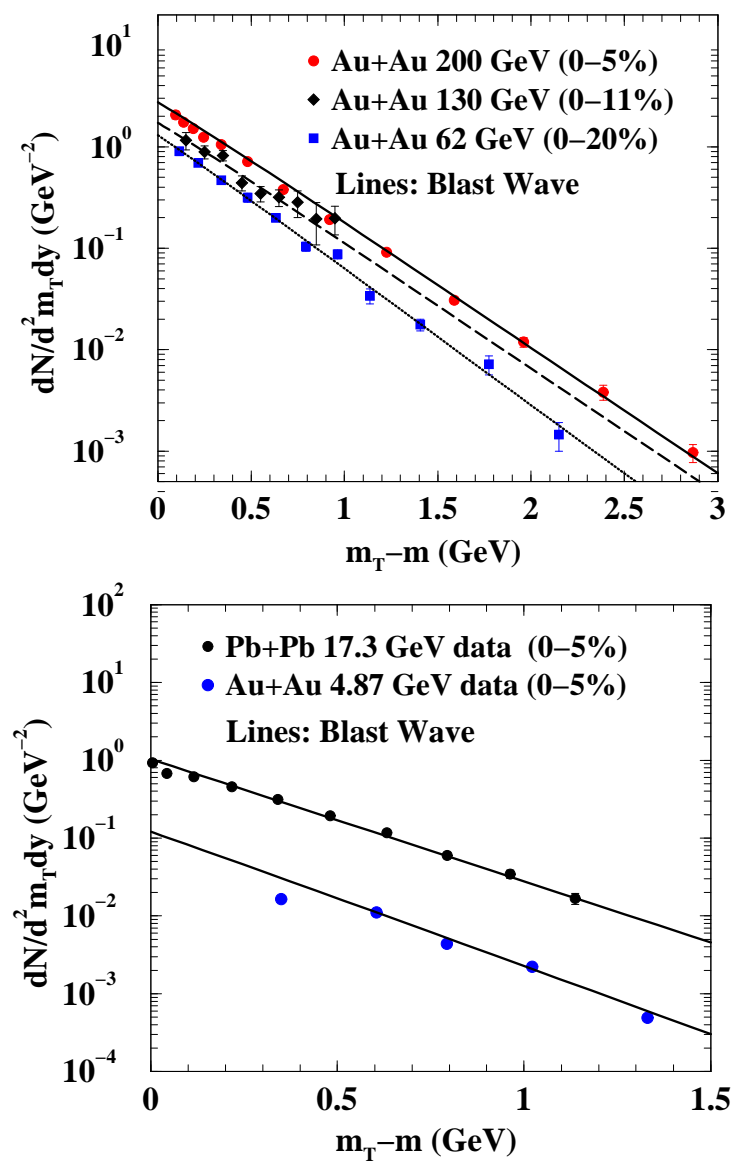

Fig. 1. The invariant yield of $\phi$ meson as a function of its kinetic energy at mid-rapidity for different colliding energies. The top figure shows the results from the STAR collaboration at RHIC. The bottom figure shows the results at SPS and AGS energies. The theoretical curves are obtained within the framework of blast wave model. 
Table 1. The table below gives the basic parameters characterizing the colliding systems at different energies, viz., centre-of-mass energy, colliding system, centrality, pseudo-rapidity density of charged particles at the central rapidity, estimated Bjorken energy density, $T_{\text {eff }}$, radial flow velocity, thermal temperature. The values of $v_{\mathrm{r}}$ are obtained from blast wave fit. The estimated error on $v_{\mathrm{r}}$ is about $10 \%$.

\begin{tabular}{ccccccccc}
\hline$\sqrt{s_{\mathrm{NN}}}(\mathrm{GeV})$ & System & Centrality & $\frac{d N_{\mathrm{ch}}}{d \eta}$ & $\epsilon_{\mathrm{Bj}}\left(\mathrm{GeV} / \mathrm{fm}^{3}\right)$ & $T_{\text {eff }}(\mathrm{GeV})$ & $v_{\mathrm{r}}$ & $T_{\mathrm{th}}(\mathrm{GeV})$ & Ref. \\
\hline 4.87 & $\mathrm{Au}-\mathrm{Au}$ & $0-5 \%$ & 120 & 1.3 & $0.196 \pm 0.02 \pm 0.02$ & 0.39 & 0.118 & $20 \mid 21]$ \\
6.3 & $\mathrm{~Pb}-\mathrm{Pb}$ & $0-7.2 \%$ & 226 & 1.0 & $0.196 \pm 0.02 \pm 0.02$ & 0.43 & 0.102 & $22 \mid 23$ \\
7.6 & $\mathrm{~Pb}-\mathrm{Pb}$ & $0-7.2 \%$ & 251 & 1.05 & $0.237 \pm 0.18 \pm 0.02$ & 0.43 & 0.143 & $22 \mid 23$ \\
8.7 & $\mathrm{~Pb}-\mathrm{Pb}$ & $0-7.2 \%$ & 272 & 1.1 & $0.244 \pm 0.09 \pm 0.06$ & 0.43 & 0.150 & $22 \mid 23$ \\
12.3 & $\mathrm{~Pb}-\mathrm{Pb}$ & $0-7.2 \%$ & 340 & 1.25 & $0.240 \pm 0.08 \pm 0.01$ & 0.43 & 0.145 & $22 \mid 23$ \\
17.3 & $\mathrm{~Pb}-\mathrm{Pb}$ & $0-5 \%$ & 426 & 2.9 & $0.299 \pm 0.07 \pm 0.01$ & 0.5 & 0.171 & $22 \mid 23$ \\
62.4 & $\mathrm{Au}-\mathrm{Au}$ & $0-20 \%$ & 472 & 3.5 & $0.328 \pm 0.06 \pm 0.02$ & 0.55 & 0.173 & 24 \\
130 & $\mathrm{Au}-\mathrm{Au}$ & $0-11 \%$ & 520 & 4.7 & $0.379 \pm 0.05 \pm 0.05$ & 0.63 & 0.176 & 24 \\
200 & $\mathrm{Au}-\mathrm{Au}$ & $0-10 \%$ & 670 & 4.9 & $0.359 \pm 0.05 \pm 0.02$ & 0.63 & 0.157 & 24 \\
200 & $\mathrm{Au}-\mathrm{Au}$ & $0-10 \%$ & 670 & 5.4 & $0.376 \pm 0.024 \pm 0.02$ & 0.63 & 0.174 & 25 \\
\hline
\end{tabular}

obtained from the BW description of the transverse momentum spectra of $\phi$. It is necessary to mention here that even at top RHIC energies, it has been observed 30 that a change of impact parameter of the collision doesn't make the required change of energy density to observe a change in phase of the produced matter (no steplike behavior in $\left\langle p_{\mathrm{T}}\right\rangle$ has been seen). So it is imperative to study the variation of temperature with energy density or center of mass energy for a fixed collision centrality.

From the experimental measurements of $d E_{\mathrm{T}} / d y$ or $d N_{\mathrm{ch}} / d y$ with $\left\langle m_{\mathrm{T}}\right\rangle, \epsilon_{\mathrm{Bj}} \cdot \tau$ can be estimated for all centralities, colliding systems and collision energies. In the absence of any accurate estimate of the formation time $\tau$, we use the quantity of $\epsilon_{\mathrm{Bj}} . \tau$. Figure 3 shows $T_{\mathrm{eff}}^{\phi}$ (top) and $T_{\mathrm{th}}^{\phi}$ (bottom) of $\phi$-mesons as functions of $\epsilon_{\mathrm{Bj}} . \tau$. In both the figures (2) and 3), the inverse slope parameter of the $p_{T}$-spectra, $T_{\text {eff }}^{\phi}$, shows an increase with collision energy/energy density till lower SPS energy is reached. A further increase in collision energy leads to an increase of early temperature and pressure of the system. As a consequence the transverse momenta of the produced hadrons and hence the inverse slope parameters increase with collision energy. This is followed by the region of constant temperature which corresponds to higher SPS energies, where it is expected that the system undergoes a deconfinement transition with the creation of a co-existing phase of partons and hadrons, which is signaled by a plateau-like structure in the above spectrum. The resulting modification of Equation of State (EoS) suppresses the transverse expansion. This observation of a plateau-like structure is equivalent to a liquid-gas phase transition with involvement of latent heat of the system, characteristic of a first order phase transition. Thus the experimental data indicate a first order phase transition, with a mixed phase stretching energy density between $\sim 1$ and $3.2 \mathrm{GeV} / \mathrm{fm}^{3}$. At higher energies (corresponding to RHIC energies), $T_{\text {eff }}^{\phi}$ further increases with collision energy. The EoS at the early stage becomes stiff again leading to increase in early pressure and temperature. The thermal component, $T_{\mathrm{th}}^{\phi}$, also shows a plateau like 


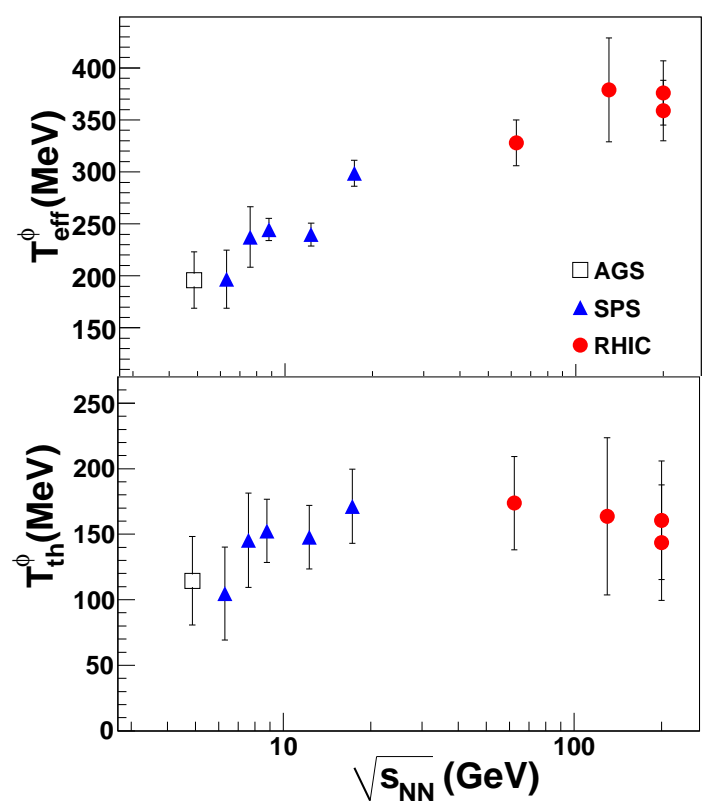

Fig. 2. Top panel shows the effective temperature of $\phi$-meson $\left(T_{\text {eff }}^{\phi}\right)$ and the bottom panel shows the $T_{\mathrm{th}}^{\phi}$ as a function of center-of-mass energy $\left(\sqrt{s_{\mathrm{NN}}}\right)$ from AGS, SPS to RHIC energies.

behavior in going toward the RHIC energies. The extraction of $T_{\mathrm{th}}^{\phi}$ involves the flow component, $v_{\mathrm{r}}$, which has an added significance. As a function of collision energy, $v_{\mathrm{r}}$ shows similar behavior as $T_{\mathrm{eff}}^{\phi} 31$. A similar pattern is suggested for the elliptic flow as a function of collision energy 32 . The observation of a mixed phase goes in line with the fact that excitation function of various observables show anomalous behavior or saturation effects starting at lower SPS energies 33|34|35|36|37. This could be related to the onset of deconfinement corresponding to SPS energy regime. In addition, the fact that microscopic transport models, based on hadronic degrees of freedom failed to reproduce the observed behavior of kaon inverse slope 3813940, also indicated the creation of a deconfinement transition corresponding to this energy regime. It has also been observed that the inverse slope parameter in $p+p$ interactions increases smoothly with collision energy with the absence of any plateau-like structure which signify a first order phase transition 36. An interesting review on onset of deconfinement in nucleus-nucleus collisions has recently been done by Gazdzicki et al. 41 . 


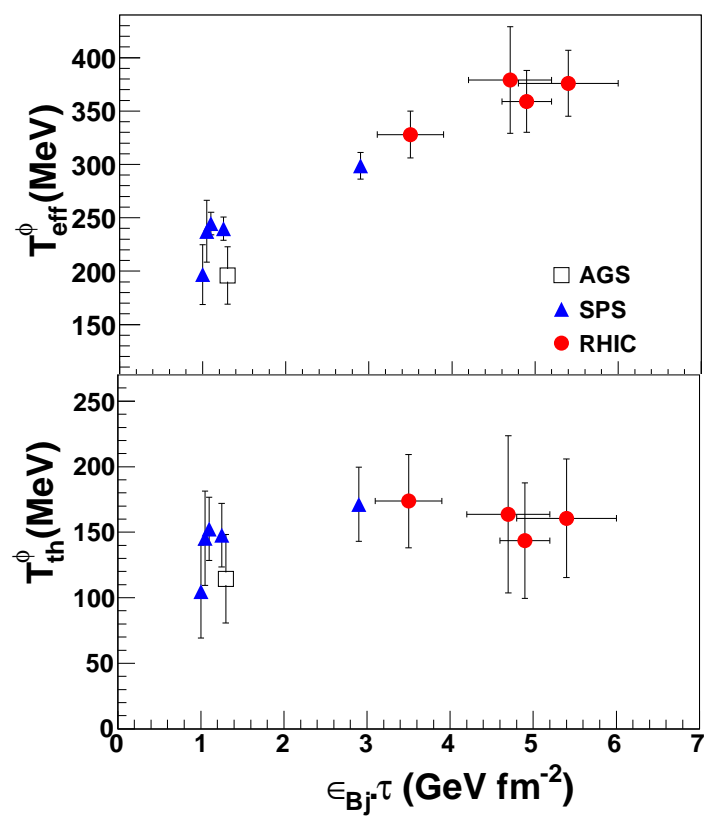

Fig. 3. Top panel shows the effective temperature of $\phi$-meson $\left(T_{\text {eff }}^{\phi}\right)$ and the bottom panel shows the $T_{\mathrm{th}}^{\phi}$ as a function of energy density multiplied to formation time $\left(\epsilon_{\mathrm{Bj}} \cdot \tau\right)$ from AGS, SPS to RHIC energies.

\section{Summary}

In summary, we observe the anomalous energy (density) dependence of transverse mass spectra of $\phi$-mesons produced in central heavy ion collisions. The inverse slope of the $m_{\mathrm{T}}$ spectrum shows an increase in AGS and RHIC energy regime, whereas it is almost constant in the intermediate SPS energies. This observation goes in line with the previous observations for lighter mesons. This anomaly could be caused by the modification of the equation of state in the transition region of the QCD deconfinement transition. The present analysis also indicates a first order phase transition stretching over an energy density of $1-3.2 \mathrm{GeV} / \mathrm{fm}^{3}$, which agrees with the lQCD prediction for the critical energy density $\left(\epsilon_{c} \sim 0.7 \mathrm{GeV} / \mathrm{fm}^{3}\right)$ for deconfinement. This observation is very exciting in view of the observed anomalies in the excitation functions of other observables, namely strangeness production, in view of the onset of deconfinement in the SPS energy domain. It would have been equally interesting to make a similar study on the spectra of $\Omega$ hyperons, as it is sensitive to the matter equation of state at the early stages of collisions. However, at present, enough data are not available for the $\Omega$ hyperons to make a similar study. The ongoing RHIC beam energy scan and the same at SPS will certainly help in getting a clear picture 
to this problem, with the advent of new data sets in the intermediate energy regime.

Acknowledgments : We thank Drs. D. Jouan, M. Floris, V. Friese, A. De Falco for providing SPS data and Profs. Y.P. Viyogi and D.P. Mahapatra for useful discussions.

\section{References}

1. F. Karsch, Prog. Part. Nucl. Phys.62, 503 (2009).

2. D.E. Miller, Phys. Rep.443, 55 (2007).

3. Z. Fodor and S. Katz, J. High Energy Phys. 04, 050 (2004).

4. C.M. Ko and D. Seibert, Phys. Rev. C 49, 2198 (1994).

5. K. Haglin, Nucl. Phys. A584, 719 (1995).

6. L. Alvarez-Ruso and V. Koch, Phys. Rev. C 65, 054901 (2002).

7. I. Kuznetsova and J. Rafelski, Phys. Rev. C 82, 035203 (2010).

8. P. Braun-Munzinger, K. Redlich and J. Stachel, Quark Gluon Plasma 3, Eds. R. C. Hwa and X. N. Wang, World Scientific (2003).

9. J.D. Bjorken, Phys. Rev. D 27, 140 (1983).

10. J. Adams et al. (STAR Collaboration), Phys. Rev. C 70, 054907 (2004).

11. Raghunath Sahoo (STAR Collaboration), Ph.D. Thesis (2007), arXiv: 0804.1800 [nuclex].

12. K. Adcox et al. (PHENIX Collaboration), Phys. Rev. Lett. 87, 052301 (2001).

13. S.S. Adler et al. (PHENIX Collaboration), Phys. Rev. C 71, 034908 (2005).

14. M. Gyulassy and T. Matsui, Phys. Rev. D 29, 419 (1984).

15. A. Dumitru and M. Gyulassy, Phys. Lett. B494, 215 (2000).

16. L. Van Hove, Phys. Lett. B118, 138 (1982).

17. B. Mohanty et al., Phys. Rev. C 68, 021901 (2003).

18. M.I. Gorenstein, M. Gazdzicki and K.A. Bugaev, Phys. Lett. B567, 175 (2003).

19. Y. Hama et al, Acta Phys. Pol. B35, 179 (2004).

20. Y. Akiba et al. (E-802 Collaboration), Phys. Rev. Lett. 76, 2021 (1996).

21. B.B. Back et al. (E917 Collaboration), Phys. Rev. C 69, 054901 (2004).

22. C. Alt et al. (NA49 Collaboration), Phys. Rev. Lett. 94, 052301 (2005).

23. C. Alt et al. (NA49 Collaboration), Phys. Rev. Lett. 78, 044907 (2008).

24. B.I. Abelev et al. (STAR Collaboration), Phys. Rev. C 79, 064903 (2009).

25. S.S. Adler et al. (PHENIX Collaboration), Phys. Rev. C 72, 014903 (2005).

26. E. Schnedermann, J. Sollfrank and U. Heinz, Phys. Rev. C 48, 2462 (1993).

27. M. Floris et al. (NA60 Collaboration), Eur. Phys. J. C49, 255 (2007).

28. M.C. Abreu et al. (NA38 Collaboration), Phys. Lett. B368, 239 (1996).

29. B. Alessandro et al. (NA50 Collaboration), Phys. Lett. B555, 147 (2003).

30. B.I. Abelev et al. (STAR Collaboration), Phys. Lett. B673, 183 (2009).

31. N. Xu, Nucl. Phys. A751, 109c (2005).

32. S.A. Voloshin, A.M. Poskanzer and R. Snellings; arXiv:0809.2949 [nucl-ex].

33. J. Cleymans et al., Phys. Lett. B660, 172 (2008).

34. J. Cleymans et al., Eur. Phys. J.: ST155, 13 (2008).

35. J. Cleymans et al., J. Phys. G: Nucl.Part. Phys.35, 104147 (2008).

36. C. Alt et al. (NA49 Collaboration), Phys. Rev. C 77, 024903 (2008).

37. S.V. Akkelin and Yu M. Sinyukov, Phys. Rev. C 73, 034908 (2006).

38. E.L. Bratkovskaya et al., Phys. Rev. C 69, 054907 (2004).

39. E.L. Bratkovskaya et al., Phys. Rev. Lett. 92, 032302 (2004).

40. M. Wagner, A.B. Larionov, and U. Mosel, Phys. Rev. C 71, 034901 (2005). 
May 12, 2022 12:20 WSPC/INSTRUCTION FILE phi-ijmpa-final

Probing the QGP Phase Boundary with Thermal Properties of $\phi$ Mesons 9

41. M. Gazdzicki et al., Acta Phys. Pol. B42, 307 (2011). 